\title{
Predictive role of the monocyte-to-lymphocyte ratio in advanced hepatocellular carcinoma patients receiving anti-PD-1 therapy
}

\author{
Zhen-Feng Zhu ${ }^{1,2,3 \#}$, Li-Ping Zhuang ${ }^{1,3 \#}$, Chen-Yue Zhang ${ }^{1,3}$, Zhou-Yu Ning ${ }^{1,3}$, Dan Wang ${ }^{1,3}$, Jie Sheng ${ }^{1,3}$, \\ Yong-Qiang Hua ${ }^{1,3}$, Jing Xie ${ }^{1,3}$, Li-Tao Xu ${ }^{1,3}$, Zhi-Qiang Meng ${ }^{1,3}$ \\ ${ }^{1}$ Department of Integrative Oncology, Fudan University Shanghai Cancer Center, Shanghai, China; ${ }^{2}$ Department of Integrative Medicine, \\ Zhongshan Hospital, Fudan University, Shanghai, China; ${ }^{3}$ Department of Oncology, Shanghai Medical College, Fudan University, Shanghai, China \\ Contributions: (I) Conception and design: ZQ Meng, LT Xu, ZF Zhu; (II) Administrative support: ZQ Meng; (III) Provision of study materials or \\ patients: YQ Hua, J Xie; (IV) Collection and assembly of data: CY Zhang, ZY Ning, D Wang, J Sheng; (V) Data analysis and interpretation: ZF Zhu, \\ LP Zhuang; (VI) Manuscript writing: All authors; (VII) Final approval of manuscript: All authors. \\ \#These authors contributed equally to this work. \\ Correspondence to: Zhi-Qiang Meng; Li-Tao Xu. Department of Integrative Oncology, Fudan University Shanghai Cancer Center, Shanghai 200032, China. \\ Email: mengshca@fudan.edu.cn; fdxlt@163.com.
}

\begin{abstract}
Background: The immune checkpoint inhibitor (ICIs) therapy has been proven effective in a range of solid tumors including hepatocellular carcinoma (HCC), non-small cell lung carcinoma and metastatic melanoma. However, only a subset of approximately $20 \%$ of patients shows an objective response to anti-PD- 1 therapy in HCC. Furthermore, the response to anti-PD-1 therapy is not correlated with programmed cell death 1 ligand expression in tumor tissue. Therefore, it is urgent to identify a biomarker to predict the response of anti-PD-1 therapy.
\end{abstract}

Methods: This retrospective study was conducted at the Fudan University Shanghai Cancer Center from December 2019 to June 2021. The monocyte-to-lymphocyte ratio (MLR) was analyzed using a receiver operating characteristic (ROC) curve. A Cox regression model and the log-rank test were used to analyze the relationship between the MLR value and the time to progression (TTP).

Results: A total of 34 advanced HCC patients were enrolled in this study. The cut-off point for the MLR at baseline was 0.35 . Univariate and multivariate Cox regression models showed that the MLR at baseline was significantly correlated with the TTP $(\mathrm{P}<0.05)$. Consistent results were found for disease progression. The log-rank test showed that patients in the low MLR group had a longer TTP $(\mathrm{P}=0.0027)$. At the time of disease progression, the median TTP in the low and high MLR groups were 33 and 18 weeks, respectively $(\mathrm{P}=0.0047)$.

Conclusions: The MLR can predict the response to anti-PD-1 therapy, and a high MLR is correlated with a short TTP in anti-PD-1-treated HCC patients.

Keywords: Hepatocellular carcinoma (HCC); anti-PD-1 therapy; monocyte-to-lymphocyte ratio (MLR); prognosis

Submitted Aug 30, 2021. Accepted for publication Nov 28, 2021.

doi: $10.21037 /$ tcr-21-1760

View this article at: https://dx.doi.org/10.21037/tcr-21-1760

\section{Introduction}

Hepatocellular carcinoma (HCC) is a common malignant tumor of the digestive system that develops rapidly and has high mortality (1). Due to the prevalence of hepatitis
$\mathrm{B}$ virus (HBV) and hepatitis $\mathrm{C}$ virus $(\mathrm{HCV})$, the incidence and mortality of HCC are currently still rising (2). Approximately $20 \%$ are diagnosed in the intermediate stage and can gain survival benefits up to 20 months from trans-arterial chemoembolization (TACE). The majority of 
patients, however, are diagnosed in the advanced stages of the disease when the disease is beyond resection and when locoregional treatments are ineffective. Thus, the prognosis for these patients is poor (3).

Recently, immune checkpoint inhibitors (ICIs) have been developed for the treatment of HCC, and nivolumab, an antibody targeting programed cell death protein 1 (PD-1), has become the third targeted drug for systemic therapy following sorafenib and regorafenib and has been overall well tolerated compared to sorafenib with low rates of grade $3 / 4$ toxicities in HCC patients (4). Nivolumab is a humanized monoclonal antibody $(\mathrm{mAb})$ that blocks the interaction between PD-1 and its ligands (PD-L1 and PD-L2) and has been approved for the treatment of advanced HCC in many countries, such as the United States (5) and Singapore. Nivolumab demonstrated significantly improved overall survival (OS) with a $58 \%$ objective response rate in advanced melanoma (6), while only approximately $20 \%$ of patients showed an objective response in HCC (4). Furthermore, the response to nivolumab was not correlated with PD-L1 expression in tumor tissue $(7,8)$. Although the tumor mutation burden (TMB) has gradually become a potential marker for predicting the efficacy of immune checkpoint treatment $(9,10)$, there are still many problems with the TMB as a predictive marker because of the high cost, the detection standards of different platforms and so on.

Systemic inflammation is associated with tumor specific immunity and studies have suggested a clinically important relationship between systemic inflammation and response to ICIs. The neutrophil-to-lymphocyte ratio (NLR), the platelet-to-lymphocyte ratio (PLR) and lymphocyte to monocyte ratio (LMR) were the commonly validated indices (11). Elevated NLR and PLR have been associated with inferior outcomes, with higher pre-treatment scores being associated with poorer OS and progression free survival (PFS) in patients treated with ICIs for non-small cell lung cancer (NSCLC) (12). A meta-analysis of 17 studies showed that patients with higher baseline NLRs had significantly poorer PFS and OS, while no significant relevance was found between PLR and clinical endpoints in patients treated with ICIs (13). LMR, as a marker of immune activation, showed an interlink with the overstimulation of the immune system. Increased LMR is associated with an unfavorable prognosis in advanced gastric cancer patients receiving immunotherapy (14). All the above research indicated that systemic inflammation is closely related to the immunotherapy response. So, whether NLR, PLR and MLR can play predictive roles in HCC patients treated with
ICIs is still unknow. For this purpose, we conduct the study.

In the present study, we aimed to identify novel biomarkers to predict the response of anti-PD-1 therapy in patients with advanced HCC. Our study showed that the monocyte-to-lymphocyte ratio (MLR) was significantly correlated with the time to progression (TTP), and patients with a high MLR showed a short TTP. Our results suggest that the MLR is a promising biomarker for predicting the response in anti-PD-1-treated advanced HCC patients.

We present the following article in accordance with the STROBE reporting checklist (available at https://tcr. amegroups.com/article/view/10.21037/tcr-21-1760/rc).

\section{Methods}

\section{Patients}

The study involving human participants were conducted in accordance with the Declaration of Helsinki (as revised in 2013). The study was approved by Institutional Ethics Board of the Fudan University Shanghai Cancer Center (IRB No. 050432-4-1212B) and individual consent for this retrospective analysis was waived. In this retrospective study, a total of 34 HCC patients who visited the Department of Integrative Oncology at Fudan University Shanghai Cancer Center between December 2019 and June 2021 were enrolled. One patient who gave up therapy after one cycle of treatment was excluded. All patients were pathologically diagnosed with HCC by tumor biopsy and received antiPD-1 therapy. All medical records, including those on age, sex, Eastern Cooperative Oncology Group (ECOG) score, $\mathrm{HBV}$ infection status, alpha-fetoprotein (AFP) level, blood cell counts, and prior therapies (including sorafenib therapy, chemotherapy and radiology), were collected with written informed consent from the enrolled patients. Blood cell counts were collected from all patients at two different time points: within 2 weeks prior to the initiation of therapy and either within 1 week when the disease progressed or 8 weeks after the initiation of therapy when the disease was stable or retracted.

Neutrophil-to-lymphocyte ratio (NLR) was calculated as absolute neutrophil count (ANC) (number of neutrophils/ $\mu \mathrm{L}$ ) divided by absolute lymphocyte count (ALC) (number of lymphocytes $/ \mu \mathrm{L}$ ), PLR was calculated as absolute platelet (PLT) count (number of PLTs/ $\mu \mathrm{L}$ ) divided by ALC (number of lymphocytes $/ \mu \mathrm{L})$, MLR was calculated as absolute monocyte count (number of PLTs/ $\mu \mathrm{L}$ ) divided by ALC (number of lymphocytes/ $\mu \mathrm{L}$ ). The above three ratios were 
compared by using receiver operating characteristic (ROC) to calculated the areas under the curve (AUC). The cutoff value of the three ratios were calculated based on the ROC curves for PFS. The best AUC of the corresponding ratio was the candidate to be a biomarker. Considering the consistence and effectiveness of biomarkers, we choose two time points (the baseline and progression) as comparison. The data at baseline is the test group, and the data after treatment is the verification group. When the results at two groups are consistent, we can make the conclusion.

\section{Treatment and evaluation}

The inclusion criteria for anti-PD-1 treatment were as follows: age $\geq 18$ years, life expectancy $\geq 4$ weeks, an ECOG performance status of 0 or 1 , the presence of advanced HCC (unresectable and/or metastatic) that was histologically confirmed, and the presence of at least one tumor lesion that could be accurately measured in at least one dimension according to the Immune-related Response Evaluation Criteria in Solid Tumors (irRECIST 1.1) (15). Previously treated lesions were not selected as target lesions. Local therapy was completed at least 4 weeks prior to the baseline scan.

A dose of $200 \mathrm{mg}$ anti-PD-1 drug was administered by a 30-minute IV infusion on day 1 of each treatment cycle every 2 weeks until progression or unacceptable toxicity. Dose reduction was performed when toxicity occurred. The median cycle of the patients is 12 weeks, range 2 to 36 weeks. Laboratory tests, including liver function tests and blood cell counts, were performed every two weeks. Follow-up with contrast-enhanced computed tomography (CT) or magnetic resonance imaging (MRI) was performed every 8 weeks after anti-PD-1 treatment to assess the tumor response and guide timely decision making for subsequent therapies. irRECIST 1.1 was used to evaluate the tumor response (15), which was classified into 4 categories: complete response (CR), partial response $(\mathrm{PR})$, stable disease, and progressive disease (PD). PD by irRECIST should be confirmed by the subsequent follow-up examination (not less than 4 weeks). Once PD occurred, other systemic or local therapies, such as chemotherapy and radiofrequency ablation, were used. The time to progression (TTP) was calculated from the date of the first treatment to the date of PD.

\section{Statistical analysis}

The cut-off value for the MLR was determined by using
ROC curves to select patients with a good response to antiPD-1 treatment. The corresponding value at the maximum sensitivity and specificity was defined as the optimal cutoff point. Paired $t$-test was used to analyze the difference between two independent samples. Cox regression models with univariate and multivariate analyses were used to identify the best predictor of survival. The predictors were estimated using the relative risk and $95 \%$ confidence intervals (CIs) in the regression model. The log-rank test was used to compare the TTP between the high and low MLR groups. Statistical significance was set at $\mathrm{P}<0.05$ (twosided). All statistical analyses were performed using SPSS software, version 19.0 (Chicago, IL, USA).

\section{Results}

\section{Baseline patient characteristics}

A total of 34 patients, including 28 men and 6 women, were enrolled in this study. The characteristics at baseline and progression are listed in Table 1. The time of baseline and progression were defined as pre-therapy and post-therapy, respectively. The mean age of the patients was 51.44 years. A total of $32 \%$ of patients had an ECOG score of 0 , and $68 \%$ of patients had an ECOG score of 1 . A total of $71 \%$ of patients had HBV infection. Moreover, $68 \%$ of patients had an AFP level of less than 400. Most patients underwent liver cancer resection (82\%). A total of $53 \%, 74 \%$ and $29 \%$ of patients received sorafenib therapy, chemotherapy and radiotherapy, respectively. The median numbers of the ANC, absolutely monocyte count (AMC), absolute lymphocyte count (ALC), PLT, NLR and PLR were 2.95, $0.5,1.40,149.5,2.4$ and 107.9, respectively.

ROC curves were used to analyze the NLR, PLR and MLR at baseline and progression to determine the best biomarker and cut-off point (Figure 1). The areas under the curve (AUC) values of the NLR, PLR and MLR at baseline were $0.569,0.711$ and 0.852 , respectively (Figure $1 A$ ). The AUC values of the NLR, PLR and MLR at progression were $0.684,0.779$ and 0.796 , respectively (Figure $1 B$ ). With the Youden index, the optimal cut-off points of the MLR at baseline and progression were both 0.35 . We chose the MLR as the optimal biomarker for survival analysis because it had the best AUC and had the same cut-off value at the two time points.

The values of the ANC, AMC, ALC, NLR, MLR and PLR at the time of baseline and progression were compared by paired $t$-test. There was no significant difference in those 
Table 1 Characteristics of anti-PD-1-treated advanced HCC patients at baseline and progression

\begin{tabular}{|c|c|c|}
\hline Variables & Pre-therapy $(n=34)$ & Post-therapy $(n=34)$ \\
\hline Age (years; mean $\pm \mathrm{SD}$ ) & $51.44 \pm 11.70$ & $51.44 \pm 11.70$ \\
\hline \multicolumn{3}{|l|}{ Gender, n } \\
\hline Female & $6(18 \%)$ & $6(18 \%)$ \\
\hline Male & $28(82 \%)$ & $28(82 \%)$ \\
\hline \multicolumn{3}{|l|}{ ECOG score, $n$} \\
\hline 0 & $11(32 \%)$ & $9(32 \%)$ \\
\hline 1 & $23(68 \%)$ & $25(68 \%)$ \\
\hline \multicolumn{3}{|l|}{ Hepatitis B infection, $n$} \\
\hline No & $10(29 \%)$ & $10(29 \%)$ \\
\hline Yes & $24(71 \%)$ & $24(71 \%)$ \\
\hline \multicolumn{3}{|l|}{ AFP, n } \\
\hline$<400 \mathrm{ng} / \mathrm{mL}$ & $23(68 \%)$ & $22(65 \%)$ \\
\hline$\geq 400 \mathrm{ng} / \mathrm{mL}$ & $11(32 \%)$ & $12(35 \%)$ \\
\hline \multicolumn{3}{|l|}{ Prior surgery, $\mathrm{n}$} \\
\hline No & $6(18 \%)$ & $6(18 \%)$ \\
\hline Yes & $28(82 \%)$ & $28(82 \%)$ \\
\hline \multicolumn{3}{|l|}{ Sorafenib, $n$} \\
\hline No & $16(47 \%)$ & $16(47 \%)$ \\
\hline Yes & $18(53 \%)$ & $18(53 \%)$ \\
\hline \multicolumn{3}{|l|}{ Prior chemotherapy, $\mathrm{n}$} \\
\hline No & $9(26 \%)$ & $9(26 \%)$ \\
\hline Yes & $25(74 \%)$ & $25(74 \%)$ \\
\hline \multicolumn{3}{|l|}{ Prior radiotherapy, $\mathrm{n}$} \\
\hline No & $24(71 \%)$ & $24(71 \%)$ \\
\hline Yes & $10(29 \%)$ & $10(29 \%)$ \\
\hline \multicolumn{3}{|l|}{ ANC, $n$} \\
\hline$<2.95 \times 10^{9} / \mathrm{L}$ & $17(50 \%)$ & $12(35 \%)$ \\
\hline$\geq 2.95 \times 10^{9 /} \mathrm{L}$ & $17(50 \%)$ & $22(65 \%)$ \\
\hline \multicolumn{3}{|l|}{$\mathrm{AMC}, \mathrm{n}$} \\
\hline$<0.5 \times 10^{9} / \mathrm{L}$ & $16(47 \%)$ & $17(50 \%)$ \\
\hline$\geq 0.5 \times 10^{9} / \mathrm{L}$ & $18(53 \%)$ & $17(50 \%)$ \\
\hline \multicolumn{3}{|l|}{ ALC, $n$} \\
\hline$<1.40 \times 10^{9} / \mathrm{L}$ & $15(44 \%)$ & $18(53 \%)$ \\
\hline$\geq 1.40 \times 10^{9} / \mathrm{L}$ & $19(56 \%)$ & $16(47 \%)$ \\
\hline
\end{tabular}

Table 1 (continued)
Table 1 (continued)

\begin{tabular}{lcc}
\hline Variables & Pre-therapy $(\mathrm{n}=34)$ Post-therapy $(\mathrm{n}=34)$ \\
\hline PLT, $\mathrm{n}$ & & \\
$<149.5 \times 10^{9} / \mathrm{L}$ & $17(50 \%)$ & $14(41 \%)$ \\
$\geq 149.5 \times 10^{9} / \mathrm{L}$ & $17(50 \%)$ & $20(59 \%)$ \\
NLR, $\mathrm{n}$ & & \\
$<2.4$ & $24(71 \%)$ & $15(44 \%)$ \\
$\geq 2.4$ & $10(29 \%)$ & $19(56 \%)$ \\
PLR, $\mathrm{n}$ & & $15(44 \%)$ \\
$<107.9$ & $22(65 \%)$ & $19(56 \%)$ \\
$\geq 107.9$ & $12(35 \%)$ & $22(65 \%)$ \\
MLR, $\mathrm{n}$ & & $12(35 \%)$ \\
$<0.35$ & $18(53 \%)$ & $41.98 \pm 4.67$ \\
$\geq 0.35$ & $16(47 \%)$ & $73.21 \pm 14.00$ \\
ALB $(\mathrm{g} / \mathrm{L} ;$ mean $\pm \mathrm{SD})$ & $43.61 \pm 4.44$ & $13.22 \pm 4.77$ \\
Cre $(\mu \mathrm{mol} / \mathrm{L} ;$ mean \pm SD) & $72.82 \pm 15.55$ & $13.22 \pm 0.73$ \\
TBIL & $13.71 \pm 6.60$ & \\
$(\mu \mathrm{mol} / \mathrm{L} ;$ mean $\pm \mathrm{SD})$ & $13.14 \pm 0.91$ & \\
PT (s; mean \pm SD) & & \\
\hline
\end{tabular}

HCC, hepatocellular carcinoma; SD, standard derivation; ECOG, Eastern Cooperative Oncology Group; AFP, alpha-fetoprotein; ANC, absolute neutrophil count; AMC, absolute monocyte count; ALC, absolute lymphocyte count; PLT, platelet; NLR, neutrophil-to-lymphocyte ratio; PLR, plate-to-lymphocyte ratio; MLR, monocyte-to-lymphocyte ratio; ALB, albumin; Cre, creatinine; TBIL, total bilirubin; PT, prothrombin time.

factors before and after therapy (Figure 2).

\section{Survival analysis}

Survival analyses including Cox regression models and logrank tests were performed to test whether the MLR could predict the response in anti-PD-1-treated HCC patients. The parameters at baseline and progression were analyzed. The median follow-up duration was 44.57 weeks, range from 13.57 to 80.86 weeks. None of the patients were lost to follow-up.

For the parameters at baseline, the gender [hazard ratio (HR): 17.121; 95\% CI: 1.57-186.686; P=0.02), ANC (HR: 0.177; 95\% CI: 0.036-0.865; $\mathrm{P}=0.032$ ) and MLR (HR: 18.693; 95\% CI: $2.962-117.994 ; \mathrm{P}=0.002$ ) were significantly correlated with the TTP in the univariate Cox regression 

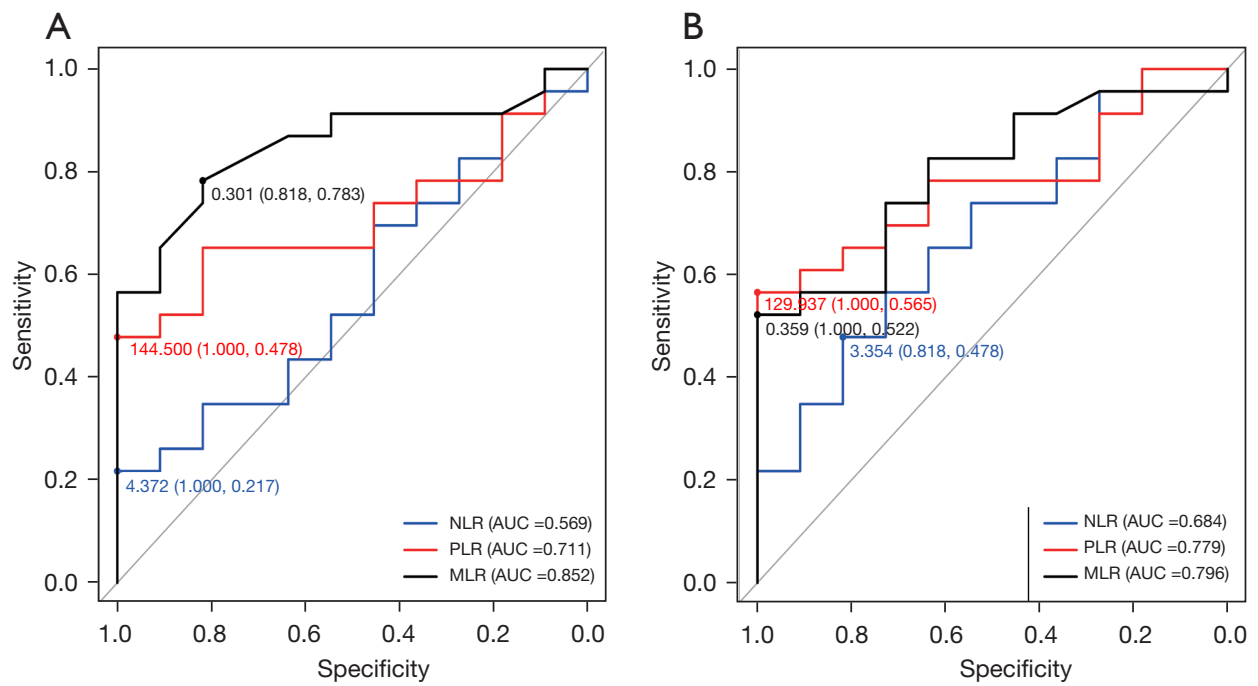

Figure 1 ROC curves were constructed to assess the specificity and sensitivity of the NLR, PLR and MLR at baseline and progression. (A) ROC curve of the NLR, PLR and MLR at baseline; the AUC were 0.569, 0.711 and 0.852, respectively. (B) ROC curve of the NLR, PLR and MLR at the time of progression; the AUC were 0.684, 0.779 and 0.796, respectively. ROC, receiver operating characteristic; NLR, neutrophil-to-lymphocyte ratio; PLR, plate-to-lymphocyte ratio; MLR, monocyte-to-lymphocyte ratio; AUC, areas under the curve.

A

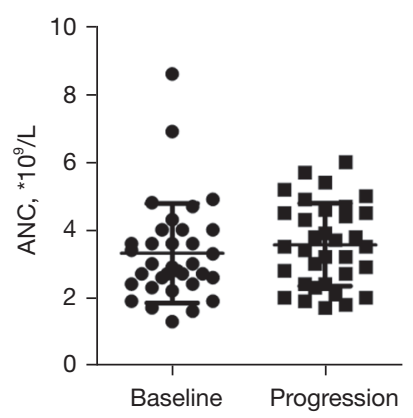

D

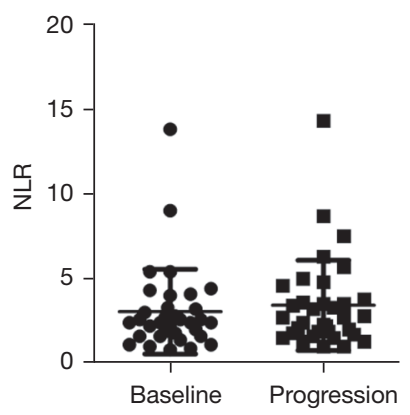

B

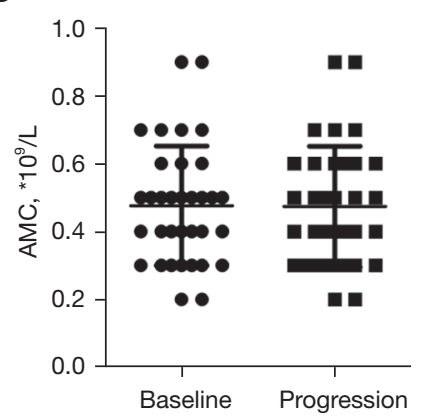

$\mathrm{E}$

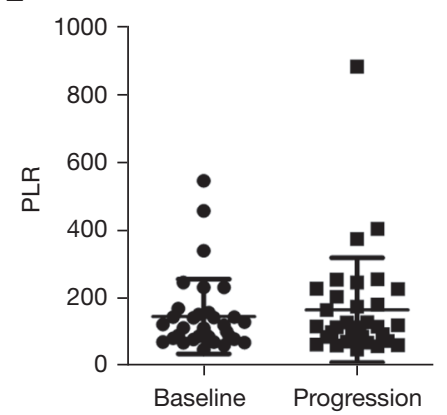

C

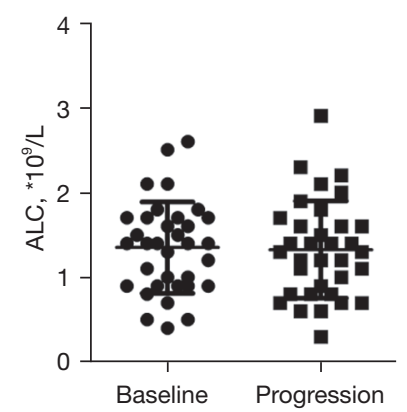

$\mathrm{F}$

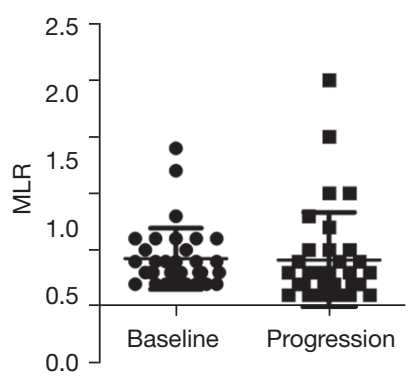

Figure 2 The index parameters, including ANC (A), AMC (B), ALC (C), NLR (D), PLR (E) and MLR (F), were analyzed by paired t-test at baseline and progression. ANC, absolute neutrophil count; AMC, absolute monocyte count; ALC, absolute lymphocyte count; NLR, neutrophil-to-lymphocyte ratio; PLR, plate-to-lymphocyte ratio; MLR, monocyte-to-lymphocyte ratio. 
Table 2 Univariate and multivariate analyses of TTP survival in 34 patients with HCC undergoing anti-PD-1 therapy at baseline

\begin{tabular}{|c|c|c|}
\hline Variables & $\mathrm{HR}(95 \% \mathrm{Cl})$ & $P$ value \\
\hline Age (years; mean \pm SD) & $0.932(0.866-1.004)$ & 0.064 \\
\hline Gender (female/male) & $17.121(1.57-186.686)$ & 0.02 \\
\hline ECOG score $(0 / 1)$ & $0.194(0.037-1.02)$ & 0.053 \\
\hline $\operatorname{AFP}(<400 \mathrm{ng} / \mathrm{mL} / \geq 400 \mathrm{ng} / \mathrm{mL})$ & 3.652 (0.587-22.734) & 0.165 \\
\hline Prior surgery (no/yes) & $0.447(0.027-7.301)$ & 0.572 \\
\hline Prior sorafenib therapy (no/yes) & $0.458(0.052-4.017)$ & 0.481 \\
\hline Prior chemotherapy (no/yes) & $0.353(0.082-1.524)$ & 0.163 \\
\hline $\operatorname{AMC}\left(<0.5 \times 10^{9} / \mathrm{L} / \geq 0.5 \times 10^{9} / \mathrm{L}\right)$ & $0.987(0.226-4.311)$ & 0.987 \\
\hline $\operatorname{ALC}\left(<1.40 \times 10^{9} / L / \geq 1.40 \times 10^{9} / L\right)$ & $2.89(0.444-18.795)$ & 0.267 \\
\hline $\operatorname{PLT}\left(<149.5 \times 10^{9} / \mathrm{L} / \geq 149.5 \times 10^{9} / \mathrm{L}\right)$ & $0.938(0.097-9.046)$ & 0.956 \\
\hline $\operatorname{NLR}(<2.4 / \geq 2.4)$ & $2.668(0.361-19.721)$ & 0.336 \\
\hline $\operatorname{PLR}(<107.9 / \geq 107.9)$ & $0.894(0.094-8.458)$ & 0.922 \\
\hline $\operatorname{MLR}(<0.35 / \geq 0.35)$ & $18.693(2.962-117.994)$ & 0.002 \\
\hline ALB (g/L; mean $\pm \mathrm{SD})$ & $0.794(0.631-1)$ & 0.05 \\
\hline Cre $(\mu \mathrm{mol} / \mathrm{L} ;$ mean $\pm \mathrm{SD})$ & $0.958(0.904-1.015)$ & 0.142 \\
\hline
\end{tabular}

TTP, time to progression; HCC, hepatocellular carcinoma; HR, hazard ratio; Cl, confidence interval; SD, standard derivation; ECOG, Eastern Cooperative Oncology Group; AFP, alpha-fetoprotein; ANC, absolute neutrophil count; AMC, absolute monocyte count; ALC, absolute lymphocyte count; PLT, platelet; NLR, neutrophil-to-lymphocyte ratio; PLR, plate-to-lymphocyte ratio; MLR, monocyte-tolymphocyte ratio; ALB, albumin; Cre, creatinine; TBIL, total bilirubin; PT, prothrombin time.

model. Multivariate Cox regression analyses were performed to avoid any interaction between these parameters. The MLR (HR: 3.455; 95\% CI: 1.442-8.279; $\mathrm{P}=0.005$ ) could serve as an independent prognostic predictor of the TTP in patients with HCC (Table 2).

A Cox regression model was also performed with the parameters at the time of progression. The univariate Cox regression analysis showed a significant correlation between the TTP and ECOG score ( $\mathrm{P}=0.047)$, AFP level $(\mathrm{P}=0.001)$, prior chemotherapy $(\mathrm{P}=0.002)$, ANC $(\mathrm{P}=0.038)$,
ALC $(\mathrm{P}=0.04)$ and MLR $(\mathrm{P}=0.003)$. The multivariate Cox regression model showed that the AFP level (HR: 5.077; 95\% CI: 1.743-14.792; $\mathrm{P}=0.003$ ), MLR (HR: 6.559; 95\% CI: 2.397-17.945; $\mathrm{P}=0.0001$ ) and creatinine (HR: 0.961; 95\% CI: 0.928-0.995; $\mathrm{P}=0.026)$ could be independent prognostic factors for the TTP (Table 3).

The Cox regression model showed that the MLR at pretherapy and post-therapy was correlated with the TTP. Therefore, a log-rank test was performed to determine the difference in the TTP between the low and high MLR 
Table 3 Univariate and multivariate analyses of TTP in 34 patients with HCC receiving anti-PD-1 therapy at the time of progression

\begin{tabular}{|c|c|c|}
\hline Variables & $\mathrm{HR}(95 \% \mathrm{Cl})$ & $P$ value \\
\hline \multicolumn{3}{|l|}{ Univariate analysis } \\
\hline Age (years; mean $\pm \mathrm{SD}$ ) & $1.084(0.997-1.18)$ & 0.059 \\
\hline Gender (female/male) & $0.944(0.025-35.684)$ & 0.975 \\
\hline ECOG score $(0 / 1)$ & $0.119(0.015-0.972)$ & 0.047 \\
\hline $\operatorname{AFP}(<400 \mathrm{ng} / \mathrm{mL} / \geq 400 \mathrm{ng} / \mathrm{mL})$ & $51.482(4.824-549.455)$ & 0.001 \\
\hline Prior surgery (no/yes) & $3.92(0.097-157.923)$ & 0.469 \\
\hline Prior sorafenib therapy (no/yes) & $6.276(0.762-51.661)$ & 0.088 \\
\hline Prior chemotherapy (no/yes) & $0.016(0.001-0.214)$ & 0.002 \\
\hline $\operatorname{AMC}\left(<0.5 \times 10^{9} / L / \geq 0.5 \times 10^{9} / \mathrm{L}\right)$ & $1.706(0.105-27.702)$ & 0.707 \\
\hline $\operatorname{ALC}\left(<1.40 \times 10^{9} / L / \geq 1.40 \times 10^{9} / L\right)$ & $111.068(1.232-10,016.629)$ & 0.04 \\
\hline $\operatorname{PLT}\left(<149.5 \times 10^{9} / \mathrm{L} / \geq 149.5 \times 10^{9} / \mathrm{L}\right)$ & $0.225(0.005-9.985)$ & 0.441 \\
\hline $\operatorname{NLR}(<2.4 / \geq 2.4)$ & $0.041(0.001-3.152)$ & 0.149 \\
\hline $\operatorname{PLR}(<107.9 / \geq 107.9)$ & $9.573(0.293-312.489)$ & 0.204 \\
\hline $\operatorname{MLR}(<0.35 / \geq 0.35)$ & $1,395.675(12.271-158,742.041)$ & 0.003 \\
\hline ALB $(g / L ;$ mean $\pm S D)$ & $1.085(0.879-1.338)$ & 0.447 \\
\hline Cre $(\mu \mathrm{mol} / \mathrm{L} ;$ mean $\pm \mathrm{SD})$ & $0.935(0.835-1.046)$ & 0.24 \\
\hline Cre $(\mu \mathrm{mol} / \mathrm{L} ;$ mean $\pm \mathrm{SD})$ & $0.961(0.928-0.995)$ & 0.026 \\
\hline
\end{tabular}

TTP, time to progression; HCC, hepatocellular carcinoma; HR, hazard ratio; Cl, confidence interval; SD, standard derivation; ECOG, Eastern Cooperative Oncology Group; AFP, alpha-fetoprotein; ANC, absolute neutrophil count; AMC, absolute monocyte count; ALC, absolute lymphocyte count; PLT, platelet; NLR, neutrophil-to-lymphocyte ratio; PLR, plate-to-lymphocyte ratio; MLR, monocyte-tolymphocyte ratio; ALB, albumin; Cre, creatinine; TBIL, total bilirubin; PT, prothrombin time.

groups. The median TTP in the low and high MLR groups at baseline was 34 and 17 weeks, respectively (HR: 0.2602; 95\% CI: 0.1080-0.6266; $\mathrm{P}=0.0027$ ) (Figure 3A). At the time of disease progression, patients with a low MLR level had a higher median TTP than those with a high MLR level (33 vs. 18 weeks, HR: 0.2544; 95\% CI: 0.0983-0.6579; $\mathrm{P}=0.0047)$ (Figure 3B).

\section{Discussion}

Inflammation is a well-known hallmark of cancer and is substantially attributed to the development and progression of malignancies (16). In solid tumors, local immune response and systemic inflammation were correlated with disease progression and patient survival $(11,17,18)$. ICIs 

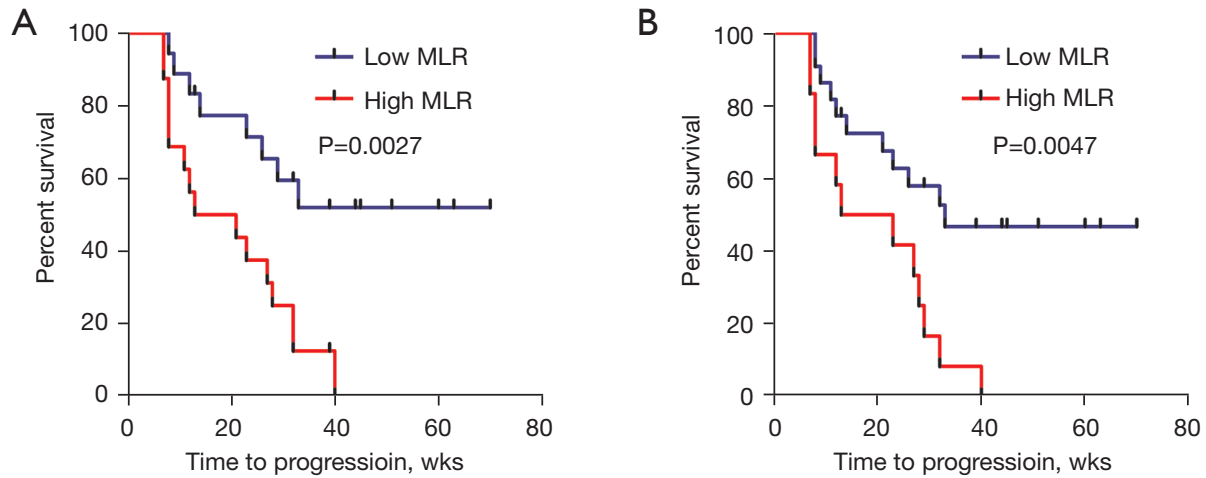

Figure 3 The TTP was compared between two groups of patients with HCC receiving anti-PD-1 therapy. Patients with a higher MLR (A) at baseline show a significantly shorter TTP. The median TTP in the low and high MLR groups at baseline was 34 and 17 weeks, respectively (HR: 0.2602; 95\% CI: 0.1080-0.6266; P=0.0027). At the time of disease progression, patients with a low MLR level had a higher median TTP than those with a high MLR level (33 vs. 18 weeks, HR: 0.2544; 95\% CI: 0.0983-0.6579; P=0.0047) (B). MLR, monocyte-tolymphocyte ratio; TTP, time to progression; HCC, hepatocellular carcinoma; HR, hazard ratio; CI, confidence interval.

are now known as an efficient therapy for patients with advanced HCC. Biomarkers that can divide patients into responders and non-responders are being explored. Because of the easy access, peripheral blood biomarkers have been researched, and the absolute blood cell count and ratios showed correlations with the drug response or OS in ICItreated patients. However, in HCC, whether peripheral blood biomarkers are correlated with the ICIs response remains unclear. Our present research enrolled 34 advanced HCC patients and is the first study to show that the MLR at treatment initiation is an independent potential biomarker for the TTP in anti-PD-1-treated HCC patients. Patients in the low MLR group showed a longer TTP than those in the high MLR group.

Circulating monocytes are recruited to the tumor stroma and differentiate into tumor-associated macrophages (TAMs). These macrophages are an important component of the tumor microenvironment and can induce the growth and progression of tumor cells by producing various cytokines and chemokines $(19,20)$. The density of TAMs was reported to correlate with the stage of disease and prognosis in solid tumors (21-23). Monocytes differentiate into macrophages, and higher monocyte counts provide a larger reservoir of macrophage precursors to influence the density of TAMs. A study indicated that peripheral AMCs were correlated with the density of TAMs in colorectal cancer (24). Low baseline AMCs predicted improved median survival after ipilimumab therapy in advanced melanoma patients (25), while the baseline AMC was not correlated with the TTP in nivolumab-treated patients with advanced HCC. Our findings highlight the possible differences in the cellular outcomes from interactions between monocytes and different tumor cell types. In response to chemotactic signals, monocytes can be polarized into macrophage subtypes (26-28). Typically, macrophages are classified into two main groups: classically activated macrophages (M1) and alternatively activated macrophages (M2) (29). M1 macrophages are pro-inflammatory cells that have potent antimicrobial activities and improve T helper 1 (Th1) cell responses; however, M2 macrophages are immunosuppressive cells supporting T helper 2 (Th2)associated effector functions $(30,31)$. Therefore, it is possible that the tumor microenvironment in anti-PD-1treated HCC patients induces monocytes to differentiate into the M2 macrophage subgroup.

In an analysis of ICI-treated patients, increasing the ALC and eosinophil count after treatment (32) or decreasing the levels of regulatory $\mathrm{T}$ cells and increasing the ALC between baseline and the end of dosing were correlated with improved survival (33). Additionally, changes in the number of absolute $\mathrm{T}$ cell counts and the absolute numbers of activated $\mathrm{T}$ cells were correlated with the clinical response (34). This finding is consistent with other analyses of patients treated with anti-PD-1 therapy, where a high ALC and ANC after 6 weeks of treatment with nivolumab were significantly associated with better survival (35). In summary, a positive association was observed between the rate of increase of the ALC and survival in previous studies, and changes in the ALC were not found to be specifically predictive of the response of anti-PD-1 therapy in our study. 
However, the MLR was correlated with the anti-PD-1treated response in HCC patients. One possible explanation for our observations involves the important role that the tumor microenvironment plays in the immune response. Cell interactions have an impact on the differentiation of monocytes and lymphocytes, so the ALCs or AMCs cannot precisely reflect the immune response of patients. Instead, the ratio of different types of immune cells might be a better reflection of the immune response than a certain type of immune cell.

To the best of our knowledge, the MLR has not been reported as a marker for treatment efficacy during immunotherapy in advanced HCC. Our data suggest the MLR as a potentially predictive biomarker in antiPD-1-treated HCC patients. However, there were a few limitations to our study. The number of enrolled patients was small, and the results need to be confirmed in a large cohort. Moreover, because of the self-limitation of retrospective studies, a prospective clinical trial needs to be conducted to further identify the role of the MLR in predicting the response to anti-PD-1 therapy.

\section{Conclusions}

The MLR can predict the response of anti-PD-1 therapy in advanced HCC patients, and a high MLR is correlated with poor prognosis in HCC patients receiving immunotherapy. It may provide useful information to oncologists in counseling of patients for immunotherapy.

\section{Acknowledgments}

Funding: This work was supported by the Leading Talents Project Foundation of Shanghai, China (grant No. 2018-045).

\section{Footnote}

Reporting Checklist: The authors have completed the STROBE reporting checklist. Available at https://tcr. amegroups.com/article/view/10.21037/tcr-21-1760/rc

Data Sharing Statement: Available at https://tcr.amegroups. com/article/view/10.21037/tcr-21-1760/dss

Peer Review File: Available at https://tcr.amegroups.com/ article/view/10.21037/tcr-21-1760/prf

Conflicts of Interest: All authors have completed the ICMJE uniform disclosure form (available at https://tcr.amegroups. com/article/view/10.21037/tcr-21-1760/coif). The authors have no conflicts of interest to declare.

Ethical Statement: The authors are accountable for all aspects of the work in ensuring that questions related to the accuracy or integrity of any part of the work are appropriately investigated and resolved. The study was conducted in accordance with the Declaration of Helsinki (as revised in 2013). The study was approved by Institutional Ethics Board of the Fudan University Shanghai Cancer Center (IRB No. 050432-4-1212B) and individual consent for this retrospective analysis was waived.

Open Access Statement: This is an Open Access article distributed in accordance with the Creative Commons Attribution-NonCommercial-NoDerivs 4.0 International License (CC BY-NC-ND 4.0), which permits the noncommercial replication and distribution of the article with the strict proviso that no changes or edits are made and the original work is properly cited (including links to both the formal publication through the relevant DOI and the license). See: https://creativecommons.org/licenses/by-nc-nd/4.0/.

\section{References}

1. Bray F, Ferlay J, Soerjomataram I, et al. Global cancer statistics 2018: GLOBOCAN estimates of incidence and mortality worldwide for 36 cancers in 185 countries. CA Cancer J Clin 2018;68:394-424.

2. Kulik L, El-Serag HB. Epidemiology and Management of Hepatocellular Carcinoma. Gastroenterology 2019;156:477-491.e1.

3. Llovet JM, Montal R, Sia D, et al. Molecular therapies and precision medicine for hepatocellular carcinoma. Nat Rev Clin Oncol 2018;15:599-616.

4. El-Khoueiry AB, Sangro B, Yau T, et al. Nivolumab in patients with advanced hepatocellular carcinoma (CheckMate 040): an open-label, non-comparative, phase 1/2 dose escalation and expansion trial. Lancet 2017;389:2492-502.

5. Nivolumab Approved for Liver Cancer. Cancer Discov 2017;7:OF3.

6. Weber J, Mandala M, Del Vecchio M, et al. Adjuvant Nivolumab versus Ipilimumab in Resected Stage III or IV Melanoma. N Engl J Med 2017;377:1824-35.

7. Robert C, Long GV, Brady B, et al. Nivolumab in previously untreated melanoma without BRAF mutation. 
N Engl J Med 2015;372:320-30.

8. Motzer RJ, Rini BI, McDermott DF, et al. Nivolumab for Metastatic Renal Cell Carcinoma: Results of a Randomized Phase II Trial. J Clin Oncol 2015;33:1430-7.

9. Allgäuer $M$, Budczies J, Christopoulos $\mathrm{P}$, et al. Implementing tumor mutational burden (TMB) analysis in routine diagnostics-a primer for molecular pathologists and clinicians. Transl Lung Cancer Res 2018;7:703-15.

10. Hellmann MD, Ciuleanu TE, Pluzanski A, et al. Nivolumab plus Ipilimumab in Lung Cancer with a High Tumor Mutational Burden. N Engl J Med 2018;378:2093-104.

11. Shelat VG. Role of inflammatory indices in management of hepatocellular carcinoma-neutrophil to lymphocyte ratio. Ann Transl Med 2020;8:912.

12. Russo A, Russano M, Franchina T, et al. Neutrophil-toLymphocyte Ratio (NLR), Platelet-to-Lymphocyte Ratio (PLR), and Outcomes with Nivolumab in Pretreated NonSmall Cell Lung Cancer (NSCLC): A Large Retrospective Multicenter Study. Adv Ther 2020;37:1145-55.

13. Tan Q, Liu S, Liang C, et al. Pretreatment hematological markers predict clinical outcome in cancer patients receiving immune checkpoint inhibitors: A meta-analysis. Thorac Cancer 2018;9:1220-30.

14. Chen Y, Zhang C, Peng Z, et al. Association of Lymphocyte-to-Monocyte Ratio With Survival in Advanced Gastric Cancer Patients Treated With Immune Checkpoint Inhibitor. Front Oncol 2021;11:589022.

15. Seymour L, Bogaerts J, Perrone A, et al. iRECIST: guidelines for response criteria for use in trials testing immunotherapeutics. Lancet Oncol 2017;18:e143-52.

16. Hanahan D, Weinberg RA. Hallmarks of cancer: the next generation. Cell 2011;144:646-74.

17. Kabir T, Ye M, Mohd Noor NA, et al. Preoperative Neutrophil-to-Lymphocyte Ratio Plus Platelet-toLymphocyte Ratio Predicts the Outcomes after Curative Resection for Hepatocellular Carcinoma. Int J Hepatol 2019;2019:4239463.

18. Nouri-Vaskeh M, Mirza-Aghazadeh-Attari M, Pashazadeh F, et al. Prognostic Impact of Monocyte to Lymphocyte Ratio in Clinical Outcome of Patients with Hepatocellular Carcinoma: A Systematic Review and Meta-analysis. Galen Med J 2020;9:e1948.

19. Mano Y, Aishima S, Fujita N, et al. Tumor-associated macrophage promotes tumor progression via STAT3 signaling in hepatocellular carcinoma. Pathobiology 2013;80:146-54.

20. Laoui D, Van Overmeire E, De Baetselier P, et al.
Functional Relationship between Tumor-Associated Macrophages and Macrophage Colony-Stimulating Factor as Contributors to Cancer Progression. Front Immunol 2014;5:489.

21. Herrera M, Herrera A, Domínguez G, et al. Cancerassociated fibroblast and M2 macrophage markers together predict outcome in colorectal cancer patients. Cancer Sci 2013;104:437-44.

22. Comito G, Giannoni E, Segura CP, et al. Cancerassociated fibroblasts and M2-polarized macrophages synergize during prostate carcinoma progression. Oncogene 2014;33:2423-31.

23. Zhang A, Qian Y, Ye Z, et al. Cancer-associated fibroblasts promote $\mathrm{M} 2$ polarization of macrophages in pancreatic ductal adenocarcinoma. Cancer Med 2017;6:463-70.

24. Shibutani M, Maeda K, Nagahara H, et al. The peripheral monocyte count is associated with the density of tumorassociated macrophages in the tumor microenvironment of colorectal cancer: a retrospective study. BMC Cancer 2017;17:404.

25. Martens A, Wistuba-Hamprecht K, Geukes Foppen M, et al. Baseline Peripheral Blood Biomarkers Associated with Clinical Outcome of Advanced Melanoma Patients Treated with Ipilimumab. Clin Cancer Res 2016;22:2908-18.

26. Lin SH, Chuang HY, Ho JC, et al. Treatment with TNF- $\alpha$ inhibitor rectifies $M 1$ macrophage polarization from blood CD14+ monocytes in patients with psoriasis independent of STAT1 and IRF-1 activation. J Dermatol Sci 2018;91:276-84.

27. Locati M, Mantovani A, Sica A. Macrophage activation and polarization as an adaptive component of innate immunity. Adv Immunol 2013;120:163-84.

28. Alvarado-Vazquez PA, Bernal L, Paige CA, et al. Macrophage-specific nanotechnology-driven CD163 overexpression in human macrophages results in an M2 phenotype under inflammatory conditions. Immunobiology 2017;222:900-12.

29. Abumaree MH, Al Jumah MA, Kalionis B, et al. Human placental mesenchymal stem cells (pMSCs) play a role as immune suppressive cells by shifting macrophage differentiation from inflammatory M1 to anti-inflammatory M2 macrophages. Stem Cell Rev Rep 2013;9:620-41.

30. Murray PJ, Wynn TA. Protective and pathogenic functions of macrophage subsets. Nat Rev Immunol 2011;11:723-37.

31. Shapouri-Moghaddam A, Mohammadian S, Vazini H, et al. Macrophage plasticity, polarization, and function in health and disease. J Cell Physiol 2018;233:6425-40.

32. Delyon J, Mateus C, Lefeuvre D, et al. Experience in daily 
practice with ipilimumab for the treatment of patients with metastatic melanoma: an early increase in lymphocyte and eosinophil counts is associated with improved survival. Ann Oncol 2013;24:1697-703.

33. Simeone E, Gentilcore G, Giannarelli D, et al. Immunological and biological changes during ipilimumab treatment and their potential correlation with clinical response and survival in patients with advanced melanoma. Cancer Immunol Immunother 2014;63:675-83.

Cite this article as: Zhu ZF, Zhuang LP, Zhang CY, Ning ZY, Wang D, Sheng J, Hua YQ, Xie J, Xu LT, Meng ZQ. Predictive role of the monocyte-to-lymphocyte ratio in advanced hepatocellular carcinoma patients receiving anti-PD-1 therapy. Transl Cancer Res 2022;11(1):160-170. doi: 10.21037/tcr-21-1760
34. Bjoern J, Juul Nitschke N, Zeeberg Iversen T, et al. Immunological correlates of treatment and response in stage IV malignant melanoma patients treated with Ipilimumab. Oncoimmunology 2016;5:e1100788.

35. Nakamura Y, Kitano S, Takahashi A, et al. Nivolumab for advanced melanoma: pretreatment prognostic factors and early outcome markers during therapy. Oncotarget 2016;7:77404-15. 